\section{Peptide scaffolds for VEGF delivery}

\section{By Kai-Jye Lou, Staff Writer}

The clinical development of VEGF-based strategies to treat ischemic cardiovascular diseases, first proposed over 10 years ago, has been challenging due to the difficulty of maintaining local VEGF concentrations at therapeutic levels. ${ }^{1,2}$ Researchers at the National Cheng Kung University in Taiwan have developed a potential solution to the problem-an injectable self-assembling peptide nanofiber scaffold that enables sustained VEGF delivery and establishes a microenvironment that augments arteriogenesis and cardiac repair following myocardial infarction. ${ }^{3}$ The group is evaluating the scaffold in porcine studies and hopes to submit an IND in two to three years.

Patrick Hsieh, an associate professor at the Institute of Clinical Medicine at the National Cheng Kung University (NCKU), said clinical trials of VEGF-based strategies to treat cardiovascular diseases have primarily relied on infusion of the protein itself or gene delivery and have not shown convincing evidence of therapeutic efficacy.

"So far, these strategies for VEGF delivery have not been very successful," he told SciBX. "And researchers in this field now realize that one cannot simply inject VEGF into the heart as it will be quickly washed out."

Moreover, newly formed blood vessels will regress within two weeks after VEGF delivery if they are not supported and maintained through maturity. ${ }^{4,5}$ A final reason to improve control over VEGF delivery and confine it to the heart is that the factor itself causes vascular leakage, which can lead to systemic side effects such as hypotension, proteinuria and edema.

Data from the largest Phase II trial of recombinant human VEGFthe investigator-led VIVA (Vascular endothelial growth factor in Ischemia for Vascular Angiogenesis) trial-showed that intracoronary and i.v. infusions of the molecule offered no improvement over placebo. ${ }^{6}$ Companies such as Vascular Genetics Inc. (now part of Madrigal Pharmaceuticals Inc.) and GenVec Inc. have previously advanced $V E G F$-based gene therapies for cardiovascular diseases as far as Phase II trials before dropping their programs.

Hsieh hypothesized that an effective delivery system for the factor needs to check three boxes: create a microenvironment that is conducive to the recruitment and engraftment of new cells in the ischemic region, establish a VEGF concentration gradient that attracts cells to the region and provide a localized, constant supply of the factor to support newly formed vasculature until it becomes mature blood vessels.

To address these challenges, Hsieh turned to an injectable selfassembling peptide nanofiber scaffold he began developing as a postdoctoral scholar at Harvard Medical School. In 2005 and 2006, Hsieh and his colleagues at Harvard reported that this self-assembling peptide scaffold could facilitate the sustained delivery of other growth factors to the heart, such as platelet derived growth factor (PDGF) and insulin-like growth factor-1 (IGF-1). ${ }^{7,8}$

The Harvard group also showed that the scaffold itself could create a microenvironment favorable to endothelial cells, which make up the inner lining of blood vessels. ${ }^{9}$

In the current study, the NCKU group combined a recombinant human VEGF isoform with a self-assembling peptide nanofiber scaffold. Over a period of 28 days, intramyocardial injection of the VEGF-containing scaffold in rat and porcine models of myocardial infarction (MI) improved cardiac function and increased both angiogenesis and arteriogenesis compared with injection of scaffold or VEGF alone.

In the rat model, intramyocardial injection of the VEGF-containing nanofiber scaffold decreased systemic adverse effects compared with injection of VEGF alone. Hsieh said the team did not see systemic or local side effects in the pig model either. Studies to evaluate the longterm effects of the VEGF-containing scaffold as well as the scaffold itself are underway.

Surprisingly, the researchers found that in addition to promoting the recruitment of cells involved in the generation of new vasculature, the VEGF-containing scaffold also promoted the recruitment of cardiomyocyte precursors. This suggests the scaffold could have the added benefit of helping generate new cardiac muscle in addition to new vasculature.

Although the experiments only evaluated effects over one month, Hsieh noted that the peptide scaffold itself degrades slowly and persists for up to four months at the injection site.

Results were published in Science Translational Medicine.

"Our scaffold maintains a consistent VEGF concentration gradient, which is important for guiding reparative endogenous cells to the injection site," said Hsieh. "Our scaffold also allows the VEGF to be retained in the heart for at least two to three weeks."

Karen Christman, a cofounder of Ventrix Inc. and an assistant professor of bioengineering at the University of California, San Diego, noted that the mechanisms underlying the therapeutic effects of engineered scaffolds for cardiac "The researchers tracked multiple cell types that are coming into the scaffold at multiple time points and related their observations to an improvement of cardiac function."

-Karen Christman,

University of California, San Diego that the current study helps to tease out such mechanisms.

"Most investigators evaluating engineered scaffolds only assess the scaffold's performance at a study's endpoint, which makes it difficult to know what is going on between the time a scaffold is implanted up until that endpoint," Christman told SciBX. "In this study, the researchers tracked multiple cell types that are coming into the scaffold at multiple time points and related their observations to an improvement of cardiac function." 
Ventrix is developing VentriGel, an injectable hydrogel scaffold sourced from decellularized porcine cardiac connective tissues, to prevent left ventricular remodeling and reduce heart failure following MI. Christman said the company hopes to move VentriGel into a Phase I trial next year.

\section{Basket of benefits}

Christman said one of the clear advantages of Hsieh's scaffold is that it is comprised of synthetic materials.

"This property gives the researchers much more control over product consistency, which will be an advantage when scaling up production of their scaffold," she told SciBX.

Michael Davis, an assistant professor of biomedical engineering and medicine at the Georgia Institute of Technology and Emory University School of Medicine, said another benefit is that the scaffold's peptides should be easy to modify if the researchers want to adjust the properties of their scaffold.

Hsieh added that many other engineered scaffolds that have been considered for use in cardiovascular disease either degrade too fast or not at all.

Scaffolds that degrade too quickly will not allow sufficient time for new blood vessels to mature, whereas long-lived, nonbiodegradable scaffolds can trigger an inflammatory response and have other long-term side effects, he told SciBX.

Hsieh thinks the scaffold being developed by his group will be able to support the generation of mature vascular networks and then get degraded and cleared from the body shortly thereafter.

He added that the scaffold provides the damaged myocardium with immediate structural support following injection, which could help prevent further ventricular remodeling and deterioration of cardiac function while reparative processes get underway.

Finally, Hsieh noted that the peptide fibers of the scaffold have diameters of 10-20 nm, which falls within the range of native extracellular matrix. He said this is an important property as it creates a microenvironment that is amenable to $3 \mathrm{D}$ cell culture.

According to Hsieh, most other biomaterials being used in engineered scaffolds for the heart form fibers in the micrometer range, which are only amenable for $2 \mathrm{D}$ cell culture. This could make it difficult to recapitulate the features of native tissues.

\section{Timing and delivery}

Hsieh said the group is running additional studies in pigs to evaluate the long-term effects of the VEGF-containing peptide scaffold.
He expects the studies to run three to six months but noted that the group might need to take the experiments out to one or two years if the FDA requires it.

In parallel, the NCKU group also is running studies to determine the therapeutic time window for delivery of the scaffold in the post-MI setting. Hsieh thinks the scaffold probably will need to be injected shortly after an acute MI event, which is when endogenous reparative processes in the heart are most active.

Davis added that it will be important to use labeled peptides to study how the scaffold degrades and where the degradation products go, and to determine whether the scaffold and any of its degradation products have significant toxicity.

Both Davis and Christman also want to know whether the scaffold could be delivered via a less-invasive route such as a catheter.

"Doctors may be hesitant to stick a needle into the heart of an acute MI patient if they've already stabilized that patient," Davis told SciBX.

NCKU has a pending patent covering the use of the peptide nanofiber scaffold for the delivery of VEGF. The technology is available for licensing.

Lou, K.-J. SciBX 5(35); doi:10.1038/scibx.2012.917

Published online Sept. 6, 2012

\section{REFERENCES}

1. Ylä-Herttuala, S. et al. J. Am. Coll. Cardiol. 49, 1015-1026 (2007)

2. von Degenfeld, G. et al. FASEB J. 20, 2657-2659 (2006)

3. Lin, Y.-D. et al. Sci. Transl. Med.; published online Aug. 8, 2012; doi:10.1126/scitranslmed.3003841

Contact: Patrick C.H. Hsieh, National Cheng Kung University Hospital, Tainan, Taiwan

e-mail: phsieh@mail.ncku.edu.tw

4. Carmeliet, P. Nat. Med. 9, 653-660 (2003)

5. Frontini, M.J. et al. Nat. Biotechnol. 29, 421-427 (2011)

6. Henry, T.D. et al. Circulation 107, 1359-1365 (2003)

7. Hsieh, P.C.H. et al. Circulation 114, 637-644 (2006)

8. Davis, M.E. et al. Proc. Natl. Acad. Sci. USA 103, 8155-8160 (2006)

9. Davis, M.E. et al. Circulation 111, 442-450 (2005)

\section{COMPANIES AND INSTITUTIONS MENTIONED}

Emory University School of Medicine, Atlanta, Ga. Georgia Institute of Technology, Atlanta, Ga. GenVec Inc. (NASDAQ:GNVC), Gaithersburg, Md. Madrigal Pharmaceuticals Inc., Fort Washington, $\mathrm{Pa}$. National Cheng Kung University, Tainan, Taiwan Harvard Medical School, Boston, Mass. University of California, San Diego, La Jolla, Calif. Ventrix Inc., San Diego, Calif. 